Vol. 3, No. 3, September 2021, pp. 225-233, doi.org:10.52567/pjsr.v3i3.244

www.pjsr.com.pk

\title{
MENTAL HEALTH PROBLEMS IN HIV AND AIDS PATIENTS LIVING IN PAKISTAN: A PRELIMINARY ANALYSIS
}

\author{
Tahira Hafeez \\ PhD Scholar, Department of Clinical Psychology, \\ University of Management and Technology, Lahore \\ tahirarubab_cp@hotmail.com \\ Zahid Mahmood \\ Department of Clinical Psychology, School of Professional Psychology, \\ University of Management and Technology, Lahore \\ zahid.mahmood@umt.edu.pk
}

\begin{abstract}
AIDS is still a burning issue in Pakistani culture, but it does not get much attention so does the mental health of PLHIVs and that is why there is no indigenous tool for the assessment of mental health problems. The present study aimed to explore mental health problems in HIV and AIDS Patients across four stages of the disease. The traditional method of scale development was adopted. The study took place in Lahore Pakistan; participants were recruited from an HIV treatment center. A list of 36 mental health problems was finalized and administered to $230 \mathrm{HIV}$-positive participants to establish the factorial structure of the scale. As a result of exploratory factor analysis, four factors (i) Anxiety, (ii) Paranoia, (iii) Elation, and (iv) Depression were identified and a scale consisted of 26 problems was finalized. The Cronbach Alpha for Mental Health Scale HIVIAIDS $(\alpha=.801)$ and GHQ-12 ( $\alpha=.79)$ show strong internal consistency and concurrent validity of the scale. All four factors have a significant correlation. One week later test-retest reliability was $r=0.40 * *(* p<.01)$ that indicated the reliability of the scale. The scale is an indigenous disease-specific assessment tool that preliminary reflects on while living in Pakistan what kind of mental health problems are faced by PLHIVs across four stages of the disease. $H I V=$ Human Immune Deficiency Virus, AIDS= Acquired Immune Deficiency Syndrome, PLHIVs= People Living with HIV and AIDS, TG= Transgender, Gay= Men having sex with men.
\end{abstract}

Keywords: HIV/AIDS, Mental Health Problems, Pakistan

\section{INTRODUCTION}

Since HIV and AIDS is a recent phenomenon, not being well explored yet, especially in Pakistan. Over the past twenty years, there have been considerable advancements in the medical treatment for HIV /AIDS, and medical treatment is provided as per international guidelines (Centers for Disease Control and Prevention, 2017; World Health Organization, 2018). But unfortunately, mental health is still ignored and unaddressed (International Federation of Red Cross and Red Crescent Societies, 2018) due to the unavailability of proper services for treatment and care. Although rigorous researches have been done across the globe on the mental health of PLHIVs, it was limited to the prevalence studies (Remien et al., 2019) of mental health problems, mainly depression and anxiety (World Health Organization, 2020). Andersen et al. (2014) focused on the subjective experience of PLHIVs related to mental health problems. Despite the limited available data in this area, some local data shows that there is a substantial burden of mental health problems like depression $(47 \%)$ and $(27.9 \%)$ anxiety was found to be prevalent among PLHIVs living in Pakistan (Hafeez, 2018; Junaid, 2021). Though literature indicates HIV/AIDS and mental problems go together, but unfortunately, there is no such specialized, culturally sensitive, and disease-specific scale available which can assess mental health problems faced by HIV/AIDS patients living in Pakistan. Scales like Beck Depression Inventory (Beck et al., 1961).

Beck Anxiety Inventory (Beck et al., 1988), and Saddique Shah Depression Scale (Siddiqui \& Shah, 1997) are existing scales. These scales are used to assess the mental health problems of HIV/AIDS 
patients. Those scales are neither culturally sensitive nor disease-specific, which generates the need for a cultural and disease-specific assessment tool. Therefore, the present study aimed to answer what mental health problems across the disease continuum are being faced by PLHIVs within the cultural context of Pakistan. And finally, to develop an indigenous, reliable, and valid assessment tool to measure and understand mental health problems PLHIVs living in Pakistan. Moreover, covering the whole spectrum of disease would lead to developing such a scale that would help to initiate timely intervention and increase treatment adherence.

\section{Research questions}

What are common mental health problems faced by PLHIVs across the disease continuum living in Pakistan?

\section{Aims and Objectives}

The proposed research would be fulfilling the following aims and objectives:

- $\quad$ The present study aimed to develop an indigenous Mental Health Scale for HIV and AIDS Patients.

- $\quad$ The study also aimed to explore mental health problems across the disease continuum.

\section{METHOD}

\section{Scale Development}

The scale development took place in three stages.

\section{Stage 1: Process of Gathering Mental Health Problems}

The first stage focused on collecting and collating mental health problems of HIV and AIDS patients across the four stages of the disease.

\section{Participants}

Participants across four stages of disease were selected to understand what kind of mental health problems PLHIVs face in the course of the disease. Eight participants (32 in total) from each stage were recruited to collect and collate mental health problems. To make the sample more inclusive, all the highrisk groups (general population, transgender, gay men, and injecting drug users), falling between 18 to 55 years of age, irrespective of gender, cognitively able to respond and understand, having no acute, active and severe infection were recruited.

\section{Collecting mental health problems}

The health care providers working at the HIV clinic were asked to refer patients fulfilling the criteria. Participants were asked, "what kind of mental health problems you are facing since you are having HIV and AIDS" and a list of mental health problems was gathered. Items with less frequency, slang, and idiosyncratic words were excluded. All the vague, dubious, and overlapping items were modified and emerged, and then a final list of 41 mental health problems was prepared (Boateng et al., 2018)

\section{Stage 2: Experts Empirical Validation of Scale}

At the second stage, empirical validation was carried out with the help of 3 psychologists working in an HIV treatment center and having at least 2 years of experience working with HIV/AIDS. Experts were requested to rate each mental health problem as per its frequency of occurrence on 4 point rating scale from $0=$ not at all, to 3 extremely common. Then the mental health problems were arranged in descending order as rated by experts. Finally, 39 mental health problems were retained and used for further exploration of psychometric properties.

\section{Pilot Study}

Mental Health Scale (MHS) consisting of 39 problems was piloted on 30 HIV/AIDS positive patients to see the comprehension of the scale. Three more items were excluded from the scale as they were not understandable for most of the participants. Finally, a list of 36 mental health problems was retained for further psychometric properties. 


\section{Stage 3: Psychometric properties}

Psychometric properties and factorial structure of the final set of statements were further established, and the scale was named as Mental Health Scale. Stratified sampling was applied (Thomas, 2020). Four stratified groups consisting of more than 50 PLHIVs for each stage of disease, i.e., stage I, stage II, stage III, and stage IV, were selected. A total of 230 participants were taken from the HIV treatment center Jinnah hospital Lahore. Moreover, the sample was calculated based on scale items (5responses against 1 item as suggested by Tabachnick et al., 2007). In the individual administration of testing protocol (MHS), each participant was asked to rate each problem on 4-point scale to the extent they face it.

\section{MEASURES}

\section{Demographic questionnaire}

The following demographics were included in the study; Registration No, Age, Gender, risk group (transgender, gay men, injecting drug user, general population), Mode of transmission: Sexual contact, blood transfusion/ blood products, unknown, Marital status (Married, Unmarried, Divorced and Separated), Education, Occupation, CD4, Treatment Status, Regimen, Medication, Regular/irregular, duration of taking ARVs, HIV status of spouse/partner, Partner disclosure and duration of disclosure.

\section{Mental Health Scale HIV/AIDS (MHS HIV/ AIDS)}

Indigenously developed Mental Health Scale HIV/AIDS (MHS HIV/AIDS) comprised of 36 items, with a 4-point rating like; "Never=0, Rarely $=1$, Sometimes $=2$, and always=3" was administered.

\section{Procedure}

The government department Punjab AIDS Control Program (PACP) was contacted to collect data. The aim of the present study was briefly shared with PACP. HIV treatment center Jinnah hospital was intimated by the PACP for the data collection, and data collection took place from October 2019 to February 2020. The researcher visited the treatment center and ensured the confidentiality of information to the authorities of the treatment center. At first, the researcher briefly shared her introduction and the purpose of the research with participants. Confidentially of information was made sure, and instructions were given in Urdu. Participants giving consent to be part of the study were given testing protocol; Demographic questionnaire and MHSHIV/AID. It took 10 minutes to complete the protocol, including 5 minutes of debriefing.

\section{RESULTS}

Descriptive and inferential data analysis was run with the SPSS 21.

Table 1: Sample Description $(n=230)$

\begin{tabular}{lll}
\hline Variables & F & Percent \\
\hline Gender(i) & 149 & 68.8 \\
Male & 35 & 15.2 \\
Female & 46 & 20 \\
Transgender & & \\
Risk Group(iii) & 64 & 27.8 \\
General population (GP) & 54 & 23.5 \\
Transgender (TG) & 56 & 24.3 \\
Gay man (MSM) & 56 & 24.3 \\
Injecting drug user (IDU) & & \\
Stages of Disease (iv) & 58 & 25.2 \\
800 and so on(1) & 56 & 24.3 \\
501-799(2) & 60 & 26.1 \\
251-500(3) & 56 & 24.3 \\
$0-250(4)$ &
\end{tabular}


I. The table represents the further description of the participants. The sample is comprised of males, females and transgender (149) $68.8 \%$, (35)15.2\%, and (46) 20 percent respectively. The major portion of sample is male dependent.

II. Basic four groups like General population (GP), Transgender (TG), Gay man (MSM) and Injecting drug user (IDU) are included as risk groups each group is sharing more than $25 \%$ of the sample. But General population (GP) 64 (27.8\%) is of the sample.

III. At least $25 \%$ sample for every stage was taken.

\section{Factor Analysis}

The mental health scale was developed to understand the mental health problems of HIV patients. The factorial structure of the mental health scale consisting of 36 items was explored by applying Principal Component analysis with Oblimin Kaiser Normalization Rotation (Kaiser, 1974), and items having loading more than 30 were retained. Furthermore, Kaiser-Meyer-Olkin measure (.89 with the $p<.001$ Bartlett's Test of Sphericity) (Stephanie, 2020) was applied to verify factor analysis as an adequate measure.

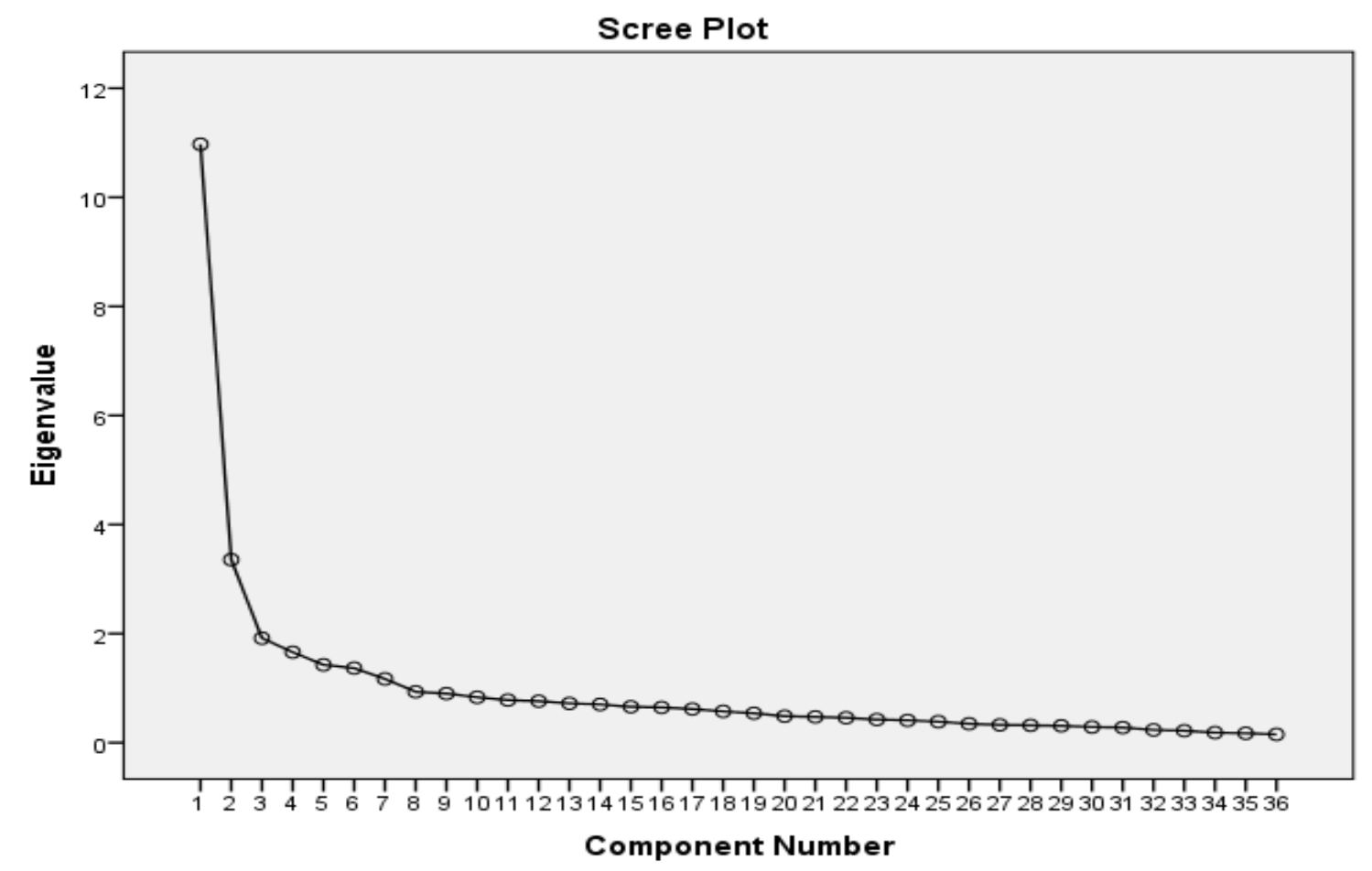

Figure 1: Scree Plot shows four factors retaining maximum number of items.

Principal Component Analysis with Oblimin Kaiser Normalization Rotation method was run on 230 participants. Total 36 items of scale were loaded. The factor loading of the scale is given below in the table. 
Table 2

Factorial presentation of Mental Health Scale (MHS HIV) with Oblimin Kaiser Normalization Rotation

\begin{tabular}{|c|c|c|c|c|}
\hline & Items & F2 & F3 & F4 \\
\hline 1 & Remain sad & & & .466 \\
\hline 2 & Remain disappointed & & & .487 \\
\hline 3 & Do not want to live & & .387 & -.302 \\
\hline 4 & Remain restless $\quad \mathbf{. 5 3 0}$ & & & \\
\hline 5 & $\begin{array}{l}\text { Do not feel like doing } .366 \\
\text { anything }\end{array}$ & & & -.315 \\
\hline 6 & Repent on mistakes & .320 & & -.384 \\
\hline 7 & Fear of getting ill & & & \\
\hline 8 & $\begin{array}{l}\text { Fear of disclosure to } \mathbf{. 6 4 4} \\
\text { family }\end{array}$ & & & \\
\hline 9 & $\begin{array}{l}\text { Fear of disclosure to } .530 \\
\text { people }\end{array}$ & & & -.325 \\
\hline 10 & Remain tired $\quad .390$ & & & -.431 \\
\hline 11 & Feeling Palpations & & & \\
\hline 12 & Fear of death & & & \\
\hline 13 & $\begin{array}{l}\text { Feeling of burden on } \mathbf{. 4 5 6} \\
\text { others }\end{array}$ & & & \\
\hline 14 & $\begin{array}{l}\text { Do not feel happiness in } \mathbf{. 3 8 6} \\
\text { anything }\end{array}$ & & & \\
\hline 15 & Fear of left alone $\quad \mathbf{. 7 4 5}$ & & & \\
\hline 16 & Felling of heart burn & & & \\
\hline 17 & Having muscle pain & & .313 & \\
\hline 18 & Worries about family & & & \\
\hline 19 & Self hatred & & .337 & \\
\hline 20 & People want to kill me & .872 & & \\
\hline 21 & $\begin{array}{l}\text { My enemy has } \\
\text { transmitted this disease to } \\
\text { me }\end{array}$ & .837 & & \\
\hline 22 & Remained angry & -.309 & & \\
\hline 23 & $\begin{array}{l}\text { Feel like taking revenge } \\
\text { from people }\end{array}$ & .638 & & \\
\hline 24 & Excessive talk & & .543 & \\
\hline 25 & $\begin{array}{l}\text { Everyone looks at me } \\
\text { with suspicion }\end{array}$ & .721 & & \\
\hline 26 & I will never be fine & & .457 & -.397 \\
\hline 27 & Have thought of suicide & & & .670 \\
\hline 28 & Do not feel energy & & & .648 \\
\hline 29 & Disturbed sleep & & .544 & \\
\hline 30 & Forgets things & & .465 & \\
\hline 31 & Attempted suicide $\quad-.343$ & & .441 & \\
\hline 32 & Feeling like life is useless & & & .762 \\
\hline 33 & $\begin{array}{l}\text { I am being punished for } \\
\text { my sins }\end{array}$ & & & .888 \\
\hline 34 & Feeling highly energetic & & .673 & \\
\hline 35 & $\begin{array}{l}\text { Having nightmares about } .322 \\
\text { disease }\end{array}$ & & .399 & .328 \\
\hline 36 & $\begin{array}{l}\text { I have been sent to } \\
\text { accomplish something big }\end{array}$ & & .504 & \\
\hline
\end{tabular}

Note: factor loadings $>.30$ have been boldfaced 
Table 2 shows items with loading. Factor analysis was run to determine the factors of MHS. Principle component analysis with Scree plot (Frey, 2018) and Oblimin with Kaiser (2016) Normalization Rotation was applied to explore the factorial structure of MHS. Based on Eigen value $>1$ factors were extracted. Two, three, four, five, and six-factor solutions were tried but a four-factor solution was found to be the best representation of the mental health problems faced by HIV and AIDS patients. In total 26 items were retained and four factors Anxiety, Paranoia, Elation, and Depression retained 10, 5, 5, and 6 items respectively. So Mental Health scale consisting of 26 problems was finalized.

\section{Factor Description}

The extracted 4 factors were given a name on the basis of item themes.

\section{Factor 1: Anxiety}

The first factor has consisted of 10 items. And items represent a common theme of apprehension and anxiousness, so the factor has been named Anxiety. The factor includes items like "fear of being exposed as HIV positive, fear of falling ill, fear of death, remained restless, fear of being left alone, and considering oneself as a burden'.

\section{Factor 2: Paranoia}

The second factor was comprised of five items, presenting the theme of paranoia. The items include "people want to kill me, my enemies transmitted this disease to me, remained angry, and everyone looks at me doubtfully".

\section{Factor 3: Elation}

Factor three retained five items reflecting the theme of Elation, keeping in view the theme, the factor was named Elation. The factor has items like" excessive talk, excessive energy, disturbed sleep, and forgets things".

\section{Factor 4: Depression}

The fourth factor clustered six items having the theme of low mood and negative feelings, so the factor was named depression. The cluster of items made of "remained sad, remained despair, suicidal thoughts, life seems worthless, and I am being punished for my sins".

Table 3: Cronbach Alpha of MHSHIV and four factors.

\begin{tabular}{lll}
\hline Factors & No of Items & A \\
\hline Anxiety & 10 & 0.75 \\
Paranoia & 5 & 0.71 \\
Elation & 5 & 0.70 \\
Depression & 6 & 0.87 \\
Total MHSHIV & 26 & 0.801 \\
\hline
\end{tabular}

The table 3 shows internal consistency of the MHSHIV. Each factor independently has high reliability as all the factors have above 0.7 Cronbach Alpha. Moreover $(\alpha=0.80)$ values indicated that all 26 items were homogenous in nature.

\section{Concurrent Validity}

The table 3 results of Cronbach Alpha Coefficient for MSHHIVAIDS ( $\alpha=.801)$ and GHQ12 ( $\alpha=79)$ shows highly significant internal consistency and concurrent validity (Frey, 2018) of scale. 


\section{Test Retest Reliability}

Furthermore, after one week, $20 \%(n=46)$ of the sample was retested to establish test retest reliability of the MHSHIV. One week later test retest reliability (Stephanie, 2020) was $\mathrm{r}=0.40^{* *}\left({ }^{*} p<.01\right)$ which indicates the reliability of the scale.

\section{DISCUSSION}

The current study aimed to explore mental health problems faced by people Living with HIV/AIDS (PLHIVs) across the disease continuum (four stages of disease). Furthermore, the study focused on the understanding of the manifestation of mental health problems with the relevance of the culture in which they manifest. Initially, a list of 41 mental health problems was gathered from 32 participants. The list of 41 collated and reported items with language arrangements was empirically validated by 3 psychologists working at an HIV treatment center. A list of 39 mental health problems was retained and further piloted on $30 \mathrm{HIV} / A I D S$ positive patients to see the comprehension of the scale. Finally, 36 mental health problems were finalized for MHS. The final list of 36 items was administered to 230 HIV/AIDS participants. With Principle component analysis, Scree plot, and Oblimin with Kaiser (1974) Normalization Rotation total of 26 items and four factors were retained. Factors were named like Anxiety, Paranoia, Elation, and Depression, each of the factors have 10, 5, 5 and 6 items respectively. So Mental Health scale consisting of 26 problems was finalized. World Health Organization (2020) and some other studies (Joska et al., 2014; Pence \& Mills, 2018) reported some of the same mental health problems like depression and anxiety as common mental health problems for PLHIVs.

The first factor has 10 problems related to fear and anxiety, PLHIVs may be overly afraid of being exposed to people as HIV-positive individuals due to stigma and discrimination attached to the disease. Being not much aware of the disease, its mode of transmission may create fear of being left alone in patients. These mental health problems might accelerate due to HIV-related stigma, discrimination, sufferings, death of a spouse, children, parents, other family members, friends, and colleagues (Joska, et al, 2014).

The second factor indicated paranoia kind of problems; it has 5 items which reflect that PLHIVs also experience paranoia along with other mental health problems, which might be due to the inflammation of virus into brain or denial. Around the globe including; United States, China, India, South Africa, several recent studies have supported the earlier study findings and identified some other mental health problems such as paranoia and psychosis (Mangurian et al., 2017; International Federation of Red Cross and Red Crescent Societies, 2018).

Elation consisted of 5 items that emerged as the next factors. Elation manifested as excessive talk, believed to be in the world to do something big. World Health Organization also identified that HIV infection not only causing psychological distress, but also impact the central nervous system, and causes neuropsychiatric complications including cognitive disorder, depression, HIV encephalopathy, mania, and dementia, and sometimes all come in one (World Health Organization, 2018; Joska et al., 2014).

The fourth factor was identified as depression, consisting of 6 problems like sadness, despair, and suicidal thoughts, similar problems have been already identified and diagnosed in PLHIVS and HIV infection itself is a highly reported factor for the risk of suicide or suicide attempts among PLHIVs (Andersen et al.,2014; Verma et al., 2017; Mangurian et al., 2017).

Since the items of the scale are direct experiences of the participants as being HIV positive patients and validated by the experts as well for the content and face validity of the scale. The results of Cronbach Alpha Coefficient for MSHHIVAIDS $(\alpha=.801)$ and GHQ12 $(\alpha=79)$ show highly significant internal consistency and concurrent validity of the scale.

The current study had a significant contribution for HIV/AIDS patients in identifying and addressing unidentified and unaddressed mental health problems. In the process of scale development through exploratory factor analysis, four factors were identified. The scale also has high internal consistency. 


\section{CONCLUSION}

The study has pointed out significant issues of PLHIVs that remain unaddressed as they prevailed unidentified. The study showed that PLHIVs not only face depression and anxiety in the course of the disease but also other mental health problems like paranoia and elation. And they are also equally important to be taken care of.

\section{Acknowledgements}

We really thank Punjab AIDS Control Program and HIV Special Clinic Jinnah hospital Lahore for their extended support.

\section{Conflict of Interest}

There is no conflict of interest among authors.

\section{Funding}

No funding was taken for this research project.

\section{REFERENCES}

Andersen, L., Kagee, A., O'Cleirigh, C., Safren, S., \& Joska, J. (2014). Understanding the experience and manifestation of depression in people living with HIV/AIDS in South Africa. AIDS care, 27(1).

Beck, A.T., Ward, C.H., Mendelson, M., Mock, J., \& Erbaugh, J. (1961). An inventory for measuring depression. Archives of General Psychiatry, 4, 561-571.

Beck, A.T., Epstein, N., Brown, G., \& Steer, R.A. (1988). An inventory for measuring clinical anxiety: Psychometric properties. Journal of Consulting and Clinical Psychology, 56(6), 893-897.

Boateng, G.O., Neilands, T.B., Frongillo, E.A., Melgar-Quiñonez, H.R., \& Young, S.L. (2018). Best Practices for Developing and Validating Scales for Health, Social, and Behavioral Research: A Primer. Front Public Health, 11(6), 149.

Centers for Disease Control and Prevention (CDC). (2017). HIV and Treatment. Retrieved from: https://www.cdc.gov/hiv/basics/whatishiv.html.

Frey, B. (2018). The SAGE Encyclopedia of educational research, measurement, and evaluation. 1-4.

Hafeez, T. (2018). A comparative study of depression and anxiety among in HIV/AIDS patients registered at treatment center in Lahore Pakistan. J Med Res Biol Stud, 1(10).

International Federation of Red Cross and Red Crescent Societies. (2018). World Disaster Report; Focus on HIV\&AIDS. Geneva, ATAR Roto Presse, Satigny/Vernier, Switzerland.

Joska, J.A., Obayemi, A.J.R, Cararra, H., \& Sorsdahl, K. (2014). Severe mental illness and retention in anti-retroviral care: A retrospective study. AIDS Behav. 18, 1492-1500.

Junaid, K., Ali, H., Khan, A.A., Khan, T.A., Khan, A.M., Khan, A., Nazim, R., \& Tahira, K. (2021). Prevalence and Associated Factors of Depression among Patients with HIV/AIDS in Lahore, Pakistan: Cross-Sectional Study. Psychology research and behavior management, 14, 77-84.

Kaiser, H.F. (1974). An index of factorial simplicity. Psychometrika. 39, 31-36.

Mangurian, C., Cournos, F., Schillinger, D., Vittinghoff, E., Creasman, J.M., \& Lee, B. (2017). Low rates of HIV testing among adults with severe mental illness receiving care in community mental health settings. Psychiatr Serv, 68, 443-448.

Pence, B.W., Mills, J.C., \& Bengtson, A.M. (2018). Association of increased chronicity of depression with HIV appointment attendance, treatment failure, and mortality among HIV-infected adults in the United States. JAMA Psychiatry, 75(4), 379-385.

Remien, R.H., Stirratt, M.J., Nguyen, N., Robbins, R.N., Pala, A.N., \& Mellins, C.A. (2019). Mental health and HIV/AIDS: the need for an integrated response. AIDS (London, England), 33(9), $1411-1420$.

Siddiqui, S., \& Shah, S.A.A. (1997). Sddiqui-Shah Depression Scale (SSDS): Development and Validation. Psychology \& Developing Societies, 2, 245-62. 
Stephanie, G. (2020). Bartlett' Test: Definition and Examples. Elementary Statistics for rest of us. Retrieved from: https://www.statisticshowto.com/bartletts-test/

Su, X., Lau, J.T., Mak, W.W., Chen, L., \& Choi, K.C. (2013). Perceived discrimination, social support, and perceived stress among people living with HIV/AIDS in China. AIDS Care, 25, 239-248.

Tabachnick, B.G., Fidell, L.S., \& Ullman, J.B. (2007). Using multivariate statistics, 5, 481-498.

Thomas, L. (2020). How to use stratified sampling. Retrieved from: https://www.scribbr. com/methodology/ stratified-sampling/

Verma, R., Pulerwitzm, J., Mahendra,V., Khandekar, S., Singh, A.K., \& Das, S. S. (2017). Promoting gender equity as a strategy to reduce HIV risk and gender-based violence among young men in India. In UNAIDS. Blind spot: Addressing a blind spot in the response to HIV. Reaching out to men and boys. 13

World Health Organization. (2018). HIV/AIDS and mental health Report.

World Health Organization. (2020). Fact sheet: Estimated number of people living with HIV/AIDS. Retrieved from: https://www.who.int/data/gho/data/indicators/indicator-details/GHO/estimatednumber-of-people-(all-ages)-living-with-hiv 